\title{
How does a combined preoperative and postoperative rehabilitation programme influence the outcome of $A C L$ reconstruction 2 years after surgery? A comparison between patients in the Delaware-Oslo ACL Cohort and the Norwegian National Knee Ligament Registry
}

\author{
H Grindem, ${ }^{1}$ L P Granan, 2,3,4 M A Risberg, ${ }^{1,5}$ L Engebretsen, 3,6 \\ L Snyder-Mackler, ${ }^{7}$ I Eitzen ${ }^{5}$
}

${ }^{1}$ Norwegian Research Center for Active Rehabilitation, Department of Sports Medicine, Norwegian School of Sport Sciences, Oslo, Norway ${ }^{2}$ Department of Physical Medicine and Rehabilitation, Oslo University Hospital, Oslo, Norway

${ }^{3}$ Oslo Sports Trauma Research Center, Norwegian School of Sport Sciences, Oslo, Norway ${ }^{4}$ Department of Pain Management and Research, Oslo University Hospital, Oslo, Norway

${ }^{5}$ Norwegian Research Center for Active Rehabilitation, Department of Orthopaedics, Oslo University Hospital, Oslo, Norway

${ }^{6}$ Department of Orthopaedics, Oslo University Hospital, Oslo, Norway

${ }^{7}$ Department of Physical Therapy, College of Health Sciences, University of Delaware, Newark, Delaware, USA

Correspondence to Dr Hege Grindem, NIMI/NAR, PB 3843 Ullevål Stadion, Oslo 0855, Norway;

hege.grindem@nih.no

Accepted 12 October 2014 Published Online First 28 October 2014

To cite: Grindem $\mathrm{H}$ Granan LP, Risberg MA, et al. Br I Sports Med 2015:49:385-389.

\section{ABSTRACT}

Background Preoperative knee function is associated with successful postoperative outcome after anterior cruciate ligament reconstruction (ACLR). However, there are few longer term studies of patients who underwent progressive preoperative and postoperative rehabilitation compared to usual care.

Objectives To compare preoperative and 2 year postoperative patient-reported outcomes (PROs) in patients undergoing progressive preoperative and postoperative rehabilitation at a sports medicine clinic compared with usual care.

Methods We included patients aged 16-40 years undergoing primary unilateral ACLR. The preoperative and 2 year postoperative Knee Injury and Osteoarthritis Outcome Score (KOOS) of 84 patients undergoing progressive preoperative and postoperative rehabilitation at a sports medicine clinic (Norwegian Research Center for Active Rehabilitation (NAR) cohort) were compared with the scores of 2690 patients from the Norwegian National Knee Ligament Registry (NKLR). The analyses were adjusted for sex, age, months from injury to surgery and cartilage/meniscus injury at ACLR.

Results The NAR cohort had significantly better preoperative KOOS in all subscales, with clinically relevant differences (>10 points) observed in KOOS Pain, activities of daily living (ADL), Sports and Quality of Life. At 2 years, the NAR cohort still had significantly better KOOS with clinically relevant differences in KOOS Symptoms, Sports and Quality of Life. At 2 years, $85.7-94 \%$ of the patients in the NAR cohort scored within the normative range of the different KOOS subscales, compared to $51.4-75.8 \%$ of the patients in the NKLR.

Conclusions Patients in a prospective cohort who underwent progressive preoperative and postoperative rehabilitation at a sports medicine clinic showed superior patient-reported outcomes both preoperatively and 2 years postoperatively compared to patients in the NKLR who received usual care.

\section{INTRODUCTION}

Rupture of the anterior cruciate ligament (ACL) is common in young, active patients. ${ }^{12}$ While a substantial proportion of injured patients undergo surgical ACL reconstruction (ACLR) to restore the mechanical stability in the knee joint, few data exist to guide how best to combine surgery and rehabilitation to optimise knee function. Given that outcomes after ACLR are not perfect, ${ }^{3}$ there is an urgent need to continue to investigate ways to provide better patient outcomes.

At our institution in Norway, acutely injured patients are recommended to undergo progressive rehabilitation for at least 5 weeks before making the decision on ACLR or continued non-surgical management. ${ }^{4-6}$ For the patients who subsequently undergo ACLR, the rationale behind this approach is that optimal preoperative knee function will lead to better postoperative knee function. ${ }^{78}$ Thus, we recommend that the patient has $90 \%$ muscle strength and hopping ability on the injured leg compared to the uninjured leg prior to ACLR. In a patient-group with no symptomatic concomitant injuries, we have previously shown significant short-term improvements in knee function and a low rate of adverse events with this approach. ${ }^{4}$ However, the longer term outcomes for those who subsequently undergo ACLR have not yet been assessed.

The Norwegian National Knee Ligament Registry (NKLR) was established in 2004, and, subsequently, several regional and national ACLR registries have been established following the same model. ${ }^{9}{ }^{10}$ These registries reflect the outcome after usual care. Data from the ACL registry can provide control material against which to compare treatment outcomes from prospective cohorts where interventions are more structured (ie, the patients in our clinic in Norway as described above).

The aim of this study was, therefore, to compare knee function preoperatively and 2 years after ACLR in patients where progressive preoperative and postoperative rehabilitation was emphasised, with patient data from the NKLR representing usual care. We hypothesised that ACLR patients who underwent progressive preoperative and postoperative rehabilitation would have higher patientreported knee function preoperatively and 2 years postoperatively compared to the patients who received usual care.

\section{METHODS}

This is a cohort study of patients undergoing either ACLR with progressive preoperative and 
postoperative active rehabilitation at a sports medicine clinic (Norwegian Research Center for Active Rehabilitation, NAR) or ACLR with usual care (NKLR). The outcome of interest is the preoperative and 2-year postoperative follow-up assessed with the Knee Injury and Osteoarthritis Outcome Score (KOOS) form. ${ }^{11} 12$

\section{Patients}

The cohort receiving the progressive preoperative and postoperative active rehabilitation programme consists of patients in the Norwegian arm of the Delaware-Oslo ACL Cohort Study. ${ }^{6}{ }^{13}$ This is a binational prospective cohort study conducted by the University of Delaware (US arm) and the NAR (Norwegian arm). These patients will therefore be referred to as the NAR cohort. Patients were consecutively screened for inclusion at the Norwegian Sports Medicine Clinic (NIMI) between 2007 and 2011. They were included in the main cohort if they had sustained a unilateral ACL rupture within the past 3 months, were between 13 and 60 years of age, and participated more than or equal to twice per week in pivoting sports. Patients were excluded if they had bilateral injuries, previous injury to either knee, or if the MRI at inclusion showed other grade III knee ligament injury, fracture or full-thickness articular cartilage injuries. Patients with meniscus injuries were excluded only if they had symptoms during plyometric activities that were not resolved within 3 months from injury. All patients were followed regardless of whether they underwent ACLR or not; however, only patients who underwent ACLR were eligible for analysis in this paper. Patients in the NAR cohort underwent ACLR at seven different hospitals and received their rehabilitation at NIMI between 2007 and 2012.

The cohort receiving usual care consists of patients recorded in the NKLR between June 2006 and December 2010. The NKLR prospectively collects data on all patients undergoing cruciate ligament surgery in Norway, including those enrolled in the NAR cohort. Further details on both cohorts have been published elsewhere. ${ }^{4} \quad 6 \quad 10 \quad 14$ Approval from the Regional Committee for Medical Research Ethics was obtained, and patients signed a written consent form prior to data collection in both cohorts.

For this study, we extracted data on all patients included in the NAR cohort or the NKLR, aged 16-40 years, undergoing primary unilateral ACLR. To ensure that the cohorts were comparable, we excluded patients who had concomitant cartilage surgery or knee ligament surgery; concomitant posterior cruciate ligament (PCL) injury, lateral collateral ligament (LCL) injury or injuries to the posterolateral corner; previous cruciate ligament injury to the contralateral knee; or previous knee surgery to the index knee. The covariates obtained to evaluate the study cohorts were sex, age, months from injury to surgery, ACL graft, cartilage injury at ACLR and meniscus injury at ACLR. These data were extracted from the NKLR. The patients in the NAR cohort were identified and extracted from the NKLR so they were not included in both cohorts.

\section{Rehabilitation}

All patients in the NAR cohort underwent a 5 -week preoperative rehabilitation programme, which has previously been described in detail, including an appendix presenting the specific exercises, progression and exercise dosage. ${ }^{4}$ The aim of the preoperative rehabilitation was to regain at least $90 \%$ quadriceps and hamstrings strength, as well as hop performance on four single-legged hop tests ${ }^{13} 1516$ prior to surgery. ${ }^{17}$ The rehabilitation programme consisted of heavy resistance strength training, plyometrics and neuromuscular exercises, and was initiated as soon as joint effusion and range of motion (ROM) deficits were resolved (mean 60.4 days after injury).

The postoperative rehabilitation was to a larger extent individually tailored based on concomitant surgery, graft source and the patient's functional status. The postoperative rehabilitation was divided into three phases. ${ }^{17}$ In the first phase (approximately 0-2 months postoperatively), the aim was to eliminate effusion, restore ROM and minimise muscular atrophy. Exercises in this phase included daily quadriceps contractions, ROM exercises and cycling as soon as tolerated. The aim of the second phase (approximately 2-6 months postoperatively) was to regain full control of weight-bearing terminal knee extension and at least $80 \%$ muscle strength and hopping ability. Neuromuscular training, strength training and plyometrics were progressively introduced during this phase. The strength training was initiated with two sets of 30 repetitions (low load) and gradually progressed to four sets of 4-6 repetitions (high load). Plyometric exercises were introduced once the strength training progressed to high load (typically 4 months postoperatively). The aim of the third phase (approximately 6-12 months postoperatively) was to regain at least $90 \%$ muscle strength and hopping ability, as well as to enable the transition to sports. This phase consisted of heavy resistance strength training and increasingly demanding plyometric exercises, as well as sport-specific drills.

\section{Outcome measures}

The patients completed the KOOS preoperatively (after preoperative rehabilitation) and 2 years postoperatively. If patients underwent an ACL revision, the 2-year KOOS from the primary ACLR was used. The KOOS is a knee-specific self-assessment instrument for knee injuries that can lead to post-traumatic osteoarthritis. The form includes 42 items in five separately scored subscales: Pain (9 items), other symptoms (7 items), function in activities of daily living (ADL; 17 items), function in sport and recreation (Sports; 5 items) and knee-related quality of life (QoL; 4 items). KOOS has previously been validated for patients undergoing ACLR. ${ }^{11} 12$ A 10 -point difference, in any subscale, was considered a clinically relevant difference. ${ }^{18}$ To detect a group difference of 10 points with an estimated SD of $20, \alpha$ level of 0.05 and $80 \%$ power, the a priori sample size calculation showed that 64 patients were needed in each group.

\section{Statistics}

Differences in group characteristics were assessed with the $\chi^{2}$ test for nominal data, the Mann-Whitney $U$ test for ordinal data and $t$ tests for interval data. Because patients with meniscus injuries were excluded from the NAR cohort only if they exhibited symptoms, a preliminary analysis was conducted to assess the interaction between the presence of meniscus injuries and cohort on all KOOS subscales, preoperatively and at 2 years. No significant interaction was found $(p \geq 0.352)$, thus the KOOS $<$ KOOS? $>$ in the two cohorts were not differently affected by meniscus injuries. Similarly, no significant interaction was found $(p \geq 0.264)$ between low-volume and high-volume hospital or injury activity $(\mathrm{p} \geq 0.138)$ and cohort on any KOOS subscale. The median number of ACLRs per hospital was 28, and a low-volume hospital was defined as hospitals with 28 surgeries or less. Injury activity was classified as being injured during sports versus other activities.

A one-way between-groups analysis of covariance (ANCOVA) was conducted to compare KOOS in the two cohorts preoperatively and 2 years postoperatively. The $\beta$ value and 95\% CI were 
reported, representing the difference in KOOS associated with the NAR protocol compared to usual care. The covariates included in the analysis were sex, age, time from injury to surgery, as well as the presence of cartilage and meniscus injury registered at ACLR. Because the NAR cohort had a significantly higher preoperative KOOS, an additional analysis of the 2 year postoperative KOOS was performed with preoperative KOOS added as a covariate. To avoid bias from ceiling effects in the NAR cohort, the analysis was stratified by the preoperative KOOS subscale score. Low/high preoperative scores were defined as scores below/above the median preoperative scores (cut-off points: Pain: 77.78, Symptoms: 75, ADL: 91.18, Sports: 45, QoL: 37.5).

The Statistical Product and Service Solutions (SPSS) for Windows, V.18.0 (SPSS, Chicago, Illinois, USA), was used to analyse the data.

To quantify the percentage of patients with KOOS within the normative range, we calculated sex-specific KOOS cut-off points for each subscale utilising the Jacobson method as described by Mann et al. ${ }^{19}$ For this method, KOOS data on a Swedish general population ${ }^{20}$ in the age group 18-34 years were used as the 'functional population' data, and the preoperative KOOS from the NKLR were used as the 'dysfunctional population' data. ${ }^{19}$

\section{RESULTS}

From the NAR cohort, 84 of 94 eligible patients completed the 2-year KOOS (89.4\%). We were unable to contact four patients,

Table 1 Patient characteristics of the NAR cohort, which underwent progressive pre-operative and postoperative rehabilitation and the NKLR cohort that represents usual care

\begin{tabular}{|c|c|c|c|}
\hline & $\begin{array}{l}\text { NAR } \\
(n=84)\end{array}$ & NKLR $(n=2690)$ & $\begin{array}{l}p \\
\text { Value }\end{array}$ \\
\hline Sex, men/women (\% men) & 39/45 (46.4) & 1362/1328 (50.6) & 0.448 \\
\hline Age, mean (SD) & $25.3(7.2)$ & $24.9(7.7)$ & 0.676 \\
\hline $\begin{array}{l}\text { Months from injury to surgery, } \\
\text { mean (SD) }\end{array}$ & $6.3(4.1)$ & $6.8(4.2)$ & 0.358 \\
\hline \multicolumn{4}{|l|}{ ACL graft, n (\%) } \\
\hline ВРТВ & $31(36.9)$ & $548(28.7)$ & 0.104 \\
\hline Hamstring & $53(63.1)$ & $1358(71.1)$ & 0.116 \\
\hline Cartilage injury, n (\%) & $10(11.9)$ & $435(16.2)$ & 0.294 \\
\hline \multicolumn{4}{|l|}{ ICRS grade } \\
\hline 1 & $3(3.6)$ & $119(4.7)$ & 0.906 \\
\hline II & $4(4.8)$ & $130(5.1)$ & \\
\hline III & $1(1.2)$ & $43(1.7)$ & \\
\hline IV & $2(2.4)$ & $8(0.3)$ & \\
\hline Meniscus injury, n (\%) & $30(35.7)$ & $857(31.9)$ & 0.456 \\
\hline Medial meniscus injury & $21(25.0)$ & $518(19.3)$ & 0.190 \\
\hline Resection & $6(7.1)$ & $257(9.6)$ & 0.458 \\
\hline Suture/fixation & $11(13.1)$ & $166(6.2)$ & 0.011 \\
\hline Untreated & $4(4.8)$ & $87(3.2)$ & 0.354 \\
\hline Lateral meniscus injury & $18(21.4)$ & $510(19.0)$ & 0.570 \\
\hline Resection & $11(13.1)$ & $324(12.0)$ & 0.771 \\
\hline Suture/fixation & $2(2.4)$ & $66(2.5)$ & 0.966 \\
\hline Untreated & $5(6.0)$ & $116(4.3)$ & 0.413 \\
\hline
\end{tabular}

two patients withdrew from the study, two patients declined to attend the follow-up and two patients had moved abroad. From the NKLR database, 5769 patients were eligible. As 3079 patients had missing data on time from injury to surgery, or missing data on all KOOS subscales at follow-up, or both, 2690 patients in the NKLR were included in the analyses (46.6\%).

Sixty-one $(72.6 \%)$ patients in the NAR cohort had achieved the preoperative functional aims. There were no significant differences between the two cohorts in age, sex, time to surgery, or presence or severity of cartilage injuries (table 1). Further, there was no significant difference in the presence of meniscus injuries; however, a higher proportion of patients in the NAR cohort had medial meniscus injuries treated with suture or fixation.

Table 2 shows the KOOS preoperatively and at 2 years for both cohorts. The NAR cohort, who had performed progressive preoperative rehabilitation, had significantly better preoperative KOOS in all subscales. The differences in all subscales except Symptoms were clinically relevant, with a group difference of 24.6 points observed in KOOS Sports. At 2 years, the NAR cohort still showed significantly better KOOS in all subscales, and clinically relevant differences were found in KOOS Symptoms, Sports and QoL. The largest group difference was still in KOOS Sports (17.7). At 2 years, the percentage of patients in the NAR cohort scoring within the normative range in the different KOOS subscales ranged from $85.7 \%$ to $94 \%$, while $51.4 \%$ to $75.8 \%$ of the patients in the NKLR scored within the normative range.

After adjusting for the preoperative KOOS, the NAR cohort had significantly better KOOS Symptoms, Sports and Recreation and QoL scores (table 3). In patients who had preoperative scores below the median score, the NAR cohort showed 20.6 higher KOOS Sports scores $(\mathrm{p}=0.003)$, and 12.3 points higher KOOS QoL scores $(p=0.006)$.

\section{DISCUSSION}

This study compared the preoperative and 2-year postoperative patient-reported knee function in patients who underwent progressive preoperative and postoperative rehabilitation at a sports medicine clinic versus usual care. The main findings of this study are the large differences in the KOOS Sports and QoL subscales, favouring the NAR cohort. These patients were recommended to regain $90 \%$ quadriceps and hamstring strength, as well as hopping performance, prior to surgery. Compared to usual care, they had superior preoperative patientreported knee function, and still exhibited superior patientreported knee function 2 years after surgery, with $86-94 \%$ of patients scoring within the normative range in the different KOOS subscales.

\section{Clinical implications}

Patients in the NAR cohort also exhibited significantly better 2-year KOOS Symptoms, Sports and QoL scores after adjusting for the preoperative scores. Clinically relevant differences were established for the patients who had low KOOS Sports and QoL preoperative scores. As the patients in the NAR cohort had preoperative KOOS that were comparable to the 2-year outcome after usual care, the lack of a large effect from surgery to 2 years postoperatively is most likely attributed to ceiling effects in the NAR cohort. The stratified analysis (table 3 ) supports this view, showing large and clinically relevant differences between the cohorts in the patients with below median preoperative KOOS Sports and QoL scores, but not in the patients with above median preoperative scores. Of the five KOOS subscales, 
Table 2 Preoperative and 2-year postoperative KOOS and percentage of patients with KOOS within the normative range

\begin{tabular}{|c|c|c|c|c|c|c|c|c|c|}
\hline \multirow[b]{2}{*}{$\begin{array}{l}\text { KOOS } \\
\text { subscale }\end{array}$} & \multirow[b]{2}{*}{ Cohort } & \multicolumn{4}{|c|}{ Preoperative } & \multicolumn{4}{|l|}{2 Years } \\
\hline & & $\begin{array}{l}\text { Mean } \\
\text { (SD) }\end{array}$ & $\begin{array}{l}\text { Percentage within } \\
\text { normative range } \\
(95 \% \mathrm{Cl})\end{array}$ & $\boldsymbol{\beta}(95 \% \mathrm{Cl})$ & p Value & $\begin{array}{l}\text { Mean } \\
\text { (SD) }\end{array}$ & $\begin{array}{l}\text { Percentage within } \\
\text { normative range } \\
(95 \% \mathrm{Cl})\end{array}$ & $\boldsymbol{\beta}(95 \% \mathrm{Cl})$ & p Value \\
\hline Pain & $\begin{array}{l}\text { NAR } \\
\text { NKLR }\end{array}$ & $\begin{array}{l}87.0(10.7) \\
75.9(16.8)\end{array}$ & $\begin{array}{l}63.1(52.8 \text { to } 73.4) \\
34.9(33.0 \text { to } 36.8)\end{array}$ & 11.5 (8.0 to 15.0$)$ & $<0.001$ & $\begin{array}{l}93.5(10.3) \\
86.0(15.1)\end{array}$ & $\begin{array}{l}89.3(82.7 \text { to } 95.9) \\
65.3(63.5 \text { to } 67.1)\end{array}$ & 7.6 (4.4 to 10.9$)$ & $<0.001$ \\
\hline Symptoms & $\begin{array}{l}\text { NAR } \\
\text { NKLR }\end{array}$ & $\begin{array}{l}82.6(12.9) \\
73.6(17.3)\end{array}$ & $\begin{array}{l}59.5(49.0 \text { to } 70.0) \\
39.6(37.6 \text { to } 41.6)\end{array}$ & 9.5 (5.8 to 13.1$)$ & $<0.001$ & $\begin{array}{l}89.2(11.9) \\
77.4(18.0)\end{array}$ & $\begin{array}{l}86.9(79.7 \text { to } 94.1) \\
51.4(49.5 \text { to } 53.3)\end{array}$ & 11.9 (8.0 to 15.8$)$ & $<0.001$ \\
\hline$A D L$ & $\begin{array}{l}\text { NAR } \\
\text { NKLR }\end{array}$ & $\begin{array}{l}94.7(10.1) \\
85.1(16.3)\end{array}$ & $\begin{array}{l}81.0(72.6 \text { to } 89.4) \\
50.5(48.5 \text { to } 52.5)\end{array}$ & $10.0(6.5$ to 13.3$)$ & $<0.001$ & $\begin{array}{l}98.0(5.6) \\
92.5(12.8)\end{array}$ & $\begin{array}{l}94.0 \text { (88.9 to } 99.1) \\
75.8 \text { (74.2 to } 77.4)\end{array}$ & 5.5 (2.8 to 8.3$)$ & $<0.001$ \\
\hline Sports & $\begin{array}{l}\text { NAR } \\
\text { NKLR }\end{array}$ & $\begin{array}{l}69.1(21.4) \\
45.2(26.6)\end{array}$ & $\begin{array}{l}61.9(51.5 \text { to } 72.3) \\
23.3(21.6 \text { to } 25.0)\end{array}$ & 24.6 (19.0 to 30.2 ) & $<0.001$ & $\begin{array}{l}85.1(16.2) \\
67.6(25.9)\end{array}$ & $\begin{array}{l}86.9(79.7 \text { to } 94.1) \\
57.2 \text { (55.3 to } 59.1)\end{array}$ & 17.7 (12.1 to 23.2$)$ & $<0.001$ \\
\hline QoL & $\begin{array}{l}\text { NAR } \\
\text { NKLR }\end{array}$ & $\begin{array}{l}49.6(20.0) \\
36.0(18.1)\end{array}$ & $\begin{array}{l}41.7(31.2 \text { to } 52.2) \\
18.2(16.6 \text { to } 19.8)\end{array}$ & 13.8 (9.9 to 17.8$)$ & $<0.001$ & $\begin{array}{l}78.6(20.4) \\
67.7(22.7)\end{array}$ & $\begin{array}{l}85.7 \text { (78.2 to } 93.2) \\
68.4 \text { (66.6 to } 70.2)\end{array}$ & 10.8 (5.9 to 15.7$)$ & $<0.001$ \\
\hline
\end{tabular}

the KOOS Sports and QoL are shown to be the most responsive and to contain the most patient-relevant items after ACLR. ${ }^{11} 21$

Several studies of preoperative determinants of successful rehabilitation after ACLR concluded that results may be optimised when muscle strength and ROM deficits are reduced. ${ }^{7} 8$ 22-24 Additionally, a recent randomised study showed that a 6 -week preoperative rehabilitation programme led to improved outcomes 12 weeks after surgery. ${ }^{25}$ All patients in the NAR cohort underwent progressive rehabilitation prior to surgery, which led to significant improvements in knee function and few adverse events. ${ }^{4}$ The current study shows that these patients not only had significantly better preoperative knee function but also superior knee function 2 years after surgery compared to usual care. Thus, the results of this study indicate that there is untapped potential for improving knee function prior to ACLR in the standard practice in Norway.

The optimal preoperative rehabilitation programme is, however, still unknown. In comparison to the study by Shaarani et $a l,{ }^{25}$ the patients in the NAR cohort followed a more intensive preoperative protocol, including perturbation training and introduction of plyometric exercises and heavy resistance strength training as soon as tolerated (approximately 2 months after injury). ${ }^{4}$ Randomised studies comparing different preoperative rehabilitation programmes are therefore needed. Note that the time from injury to surgery was not different between the NAR cohort $(6.3 \pm 4.1$ months) and the NKLR $(6.8 \pm 4.2$ months), indicating that the preoperative rehabilitation did not entail postponing the date of surgery for these patients.

\section{Why did the outcomes differ between groups?}

We acknowledge that being treated at a sports medicine clinic may include benefits over and above the rehabilitation protocol itself. It is likely that patient education and psychological strategies utilised by the doctors and physiotherapists treating the NAR cohort differ from usual care. Clinicians treating the NAR cohort placed heavy emphasis on educating the patients about their injury, the ACLR procedure and the importance of rehabilitation. The rehabilitation focus was to regain knee function and muscle strength using functional milestones and repeated testing prior to surgery, and at 6 months, 1 year and 2 years postoperatively. Thus, we believe these patients benefitted from more comprehensive follow-ups and clearer goal setting than usual care. While offering emotional and listening support is considered a natural part of the clinicians' jobs, no standardised form of cognitive behavioural therapy was used and none of the patients were referred to sports psychologists. However, such an intense rehabilitation programme could worsen patient outcomes if the knee was inherently unstable.

Table 3 Difference in 2-year postoperative KOOS in patients with below and above median preoperative scores after adjustment for preoperative KOOS

\begin{tabular}{|c|c|c|c|c|c|c|c|c|c|c|}
\hline & \multirow{2}{*}{\multicolumn{2}{|c|}{ All patients }} & \multicolumn{4}{|c|}{ Low preoperative score } & \multicolumn{4}{|c|}{ High preoperative score } \\
\hline & & & \multicolumn{2}{|l|}{$\mathrm{N}$} & \multirow[b]{2}{*}{$\boldsymbol{\beta}(95 \% \mathrm{Cl})$} & \multirow[b]{2}{*}{ pValue } & \multicolumn{2}{|l|}{$\mathbf{N}$} & \multirow[b]{2}{*}{$\boldsymbol{\beta}(95 \% \mathrm{Cl})$} & \multirow[b]{2}{*}{$p$ Value } \\
\hline & $\boldsymbol{\beta}(95 \% \mathrm{Cl})$ & $p$ Value & NAR & NKLR & & & NAR & NKLR & & \\
\hline Pain & 2.9 (0.0 to 5.8$)$ & 0.053 & 12 & 1059 & $3.4(-5.9$ to 12.6$)$ & 0.472 & 72 & 1296 & $2.9(0.4$ to 5.3$)$ & 0.023 \\
\hline Symptoms & 8.5 (4.9 to 12.2 ) & $<0.001$ & 33 & 1270 & $6.2(-0.1$ to 12.5$)$ & 0.053 & 51 & 1123 & 9.7 (5.4 to 13.9$)$ & $<0.001$ \\
\hline ADL & $2.3(-0.1$ to 4.8$)$ & 0.065 & 16 & 1271 & $4.5(-2.0$ to 11.0$)$ & 0.173 & 68 & 1085 & $1.5(-0.7$ to 3.7$)$ & 0.191 \\
\hline Sports & 7.6 (2.4 to 12.7$)$ & 0.004 & 14 & 1253 & 20.6 (7.0 to 34.1$)$ & 0.003 & 70 & 1088 & $5.1(0.2$ to 10.0$)$ & 0.042 \\
\hline QoL & 5.5 (0.8 to 10.2$)$ & 0.022 & 27 & 1477 & 12.3 (3.6 to 21.0$)$ & 0.006 & 57 & 894 & $2.1(-2.9$ to 7.2$)$ & 0.409 \\
\hline
\end{tabular}

$\beta$ Represents the difference in KOOS associated with the NAR cohort versus the NKLR after adjustment for preoperative KOOS, sex, age, time from injury to surgery, meniscus injury at ACLR and cartilage injury at ACLR. Positive values indicate higher scores in the NAR cohort.

Low/high preoperative scores are defined as scores below/above the median preoperative scores (cut-off points: Pain: 77.78, Symptoms: 75, ADL: 91.18, Sports: 45, QoL: 37.5).

ACLR, anterior cruciate ligament reconstruction; ADL, activities of daily living; KOOS, Knee Injury and Osteoarthritis Outcome Score; NAR, Norwegian Research Center for Active Rehabilitation; NKLR, Norwegian National Knee Ligament Registry; QoL, quality of life. 
We believe our study addressed a question that had equipoisedoes intense rehabilitation benefit or harm patients who may undergo ACLR.

The major limitation of this study is that the study design does not allow us to solidly infer about causality, that is, whether it was the rehabilitation or other unmeasured factors that caused superior outcomes in the NAR cohort. Another limitation is the lack of KOOS early after injury. Although the short-term benefit of the preoperative rehabilitation in the NAR cohort has been shown in a previous publication, ${ }^{4}$ the NKLR does not have access to patient outcomes in the period between injury and surgery. The patients in the NAR cohort may be hypothesised to be more motivated for rehabilitation or to have a higher socioeconomic level than the general population. However, the NKLR comprises patients from all sports medicine clinics, and the treatment expenses for the patients in the NAR cohort were covered by the public healthcare system (expenses for surgery as well as rehabilitation for 6 months after injury and 6 months after surgery), or by compulsory sports insurance. Further, only two patients $(1.2 \%$ per year) in the NAR cohort underwent revision surgery, which is comparable to the previously reported annual revision rate of $0.9 \%$ in the NKLR. ${ }^{9}$ The major strength of this study is the robustness of the outcome data from the NKLR due to the large number of patients. However, a large percentage of the patients had missing data. The baseline or 2-year KOOS did not differ in any relevant way between patients with complete data and patients with partially complete data (mean differences ranging from 1.3 to 4.7 in favour of patients with complete data), nor was there any relevant difference in demographics, concomitants or surgical data (data not shown). Furthermore, the NAR cohort is included from a prospective study with an $89.4 \%$ follow-up rate, benefitting from more continuous and comprehensive evaluation than what is common in registry studies. It is also relevant that this study evaluates outcomes of the largest patient group that experiences ACL injuries and receives ACLR-young active patients of both sexes.

\section{CONCLUSION}

ACLR patients, aged 16-40 years, who followed a progressive preoperative and postoperative active rehabilitation programme at a sports medicine clinic showed superior 2-year postoperative patient-reported outcomes compared with patients who underwent usual care. Implementation of this treatment strategy will potentially enable the patient to regain knee function 2 years postoperatively that is comparable to the general population.

\section{What are the new findings?}

- This is the first study to utilise anterior cruciate ligament reconstruction (ACLR) registry data to compare treatment outcomes following usual care with a prospective cohort who underwent progressive preoperative and postoperative rehabilitation at a sports medicine clinic.

- ACLR patients who underwent progressive preoperative and postoperative active rehabilitation at the sports medicine clinic showed superior 2-year patient-reported outcomes compared with usual care.

- $86-94 \%$ of the ACLR patients who underwent progressive preoperative and postoperative rehabilitation at the sports medicine clinic had 2-year postoperative patient-reported outcomes comparable to the general population.

\section{How might it impact on clinical practice in the near future?}

- The treatment strategy described in this paper, including progressive preoperative and postoperative active rehabilitation and comprehensive functional testing, should be considered in the standard treatment protocol of patients who undergo ACLR.

- Based on our previous findings and the 2-year results in this paper, an intensive preoperative rehabilitation protocol including heavy resistance strength training and plyometrics should be considered beneficial, and not harmful, as long as functional criteria for initiation of exercises are met.

- Future studies are needed to identify which parts of the described treatment strategy are the key factors responsible for the observed differences compared to usual care.

Acknowledgements The authors thank physiotherapists Annika Storevold, Ida Svege, Håvard Moksnes, Espen Selboskar, Karin Rydevik and Marte Lund for their assistance in data collection for this study. They acknowledge the Norwegian Sports Medicine Clinic, NIMI (http://www.nimi.no), for supporting the Norwegian Research Center for Active Rehabilitation (NAR; http://www.active-rehab.no) with rehabilitation facilities and research staff.

Contributors All authors contributed to the conception and design, interpretation of the data, critical revision and final approval of the article. IE, HG and LPG were responsible for data acquisition; HG and LPG were responsible for data analysis.

\section{Competing interests None.}

Patient consent Obtained.

Ethics approval The Regional Committee for Medical Research Ethics in South Eastern Norway.

Provenance and peer review Not commissioned; externally peer reviewed.

\section{REFERENCES}

1 Hewett TE, Di Stasi SL, Myer GD. Current concepts for injury prevention in athletes after anterior cruciate ligament reconstruction. Am J Sports Med 2013;41:216-24.

2 Spindler KP, Wright RW. Clinical practice. Anterior cruciate ligament tear. N Eng/ J Med 2008;359:2135-42.

3 Ardern CL, Taylor NF, Feller JA, et al. Fifty-five per cent return to competitive sport following anterior cruciate ligament reconstruction surgery: an updated systematic review and meta-analysis including aspects of physical functioning and contextual factors. Br J Sports Med 2014;48:1543-52.

4 Eitzen I, Moksnes H, Snyder-Mackler L, et al. A progressive 5-week exercise therapy program leads to significant improvement in knee function early after anterior cruciate ligament injury. J Orthop Sports Phys Ther 2010;40:705-21.

5 Grindem H, Eitzen I, Moksnes $\mathrm{H}$, et al. A pair-matched comparison of return to pivoting sports at 1 year in anterior cruciate ligament-injured patients after a nonoperative versus an operative treatment course. Am J Sports Med 2012;40:2509-16.

6 Grindem H, Eitzen I, Engebretsen L, et al. Nonsurgical or surgical treatment of $A C L$ injuries: knee function, sports participation, and knee reinjury: the Delaware-Oslo ACL cohort study. J Bone Joint Surg Am 2014;96:1233-41.

7 Eitzen I, Holm I, Risberg MA. Preoperative quadriceps strength is a significant predictor of knee function two years after anterior cruciate ligament reconstruction. Br J Sports Med 2009;43:371-6.

8 de Jong SN, van Caspel DR, van Haeff MJ, et al. Functional assessment and muscle strength before and after reconstruction of chronic anterior cruciate ligament lesions. Arthroscopy 2007;23:21-8, 28.e1-3.

9 Maletis GB, Granan LP, Inacio MC, et al. Comparison of community-based ACL reconstruction registries in the U.S. and Norway. J Bone Joint Surg Am 2011;93 (Suppl 3):31-6.

10 Granan LP, Forssblad M, Lind M, et al. The Scandinavian ACL registries 20042007: baseline epidemiology. Acta Orthop 2009;80:563-7.

11 Roos EM, Roos HP, Lohmander LS, et al. Knee Injury and Osteoarthritis Outcome Score (KOOS) - development of a self-administered outcome measure. J Orthop Sports Phys Ther 1998;28:88-96.

12 Roos EM, Roos HP, Ekdahl C, et al. Knee injury and Osteoarthritis Outcome Score (KOOS)—validation of a Swedish version. Scand J Med Sci Sports 1998;8:439-48. 


\section{Original article}

13 Logerstedt D, Grindem H, Lynch A, et al. Single-legged hop tests as predictors of self-reported knee function after anterior cruciate ligament reconstruction: the Delaware-Oslo ACL cohort study. Am J Sports Med 2012;40:2348-56.

14 Granan LP, Bahr R, Steindal K, et al. Development of a national cruciate ligament surgery registry: the Norwegian National Knee Ligament Registry. Am I Sports Med 2008:36:308-15.

15 Noyes FR, Barber SD, Mangine RE. Abnormal lower limb symmetry determined by function hop tests after anterior cruciate ligament rupture. Am I Sports Med 1991;19:513-18.

16 Grindem H, Logerstedt D, Eitzen I, et al. Single-legged hop tests as predictors of self-reported knee function in nonoperatively treated individuals with anterior cruciate ligament injury. Am I Sports Med 2011;39:2347-54.

17 Eitzen I. Rehabilitering efter rekonstruktion af forreste korsbånd. Fysioterapeuten 2011;1:8-19.

18 Roos EM, Lohmander LS. The Knee injury and Osteoarthritis Outcome Score (KOOS): from joint injury to osteoarthritis. Health Qual Life Outcomes 2003;1:64.

19 Mann BJ, Gosens T, Lyman S. Quantifying clinically significant change: a brief review of methods and presentation of a hybrid approach. Am I Sports Med 2012;40:2385-93.
20 Paradowski PT, Bergman S, Sunden-Lundius A, et al. Knee complaints vary with age and gender in the adult population. Population-based reference data for the Knee injury and Osteoarthritis Outcome Score (KOOS). BMC Musculoskelet Disord 2006;7:38.

21 Hambly K, Griva K. IKDC or KOOS: which one captures symptoms and disabilities most important to patients who have undergone initial anterior cruciate ligament reconstruction? Am I Sports Med 2010;38:1395-404.

22 de Valk EJ, Moen MH, Winters M, et al. Preoperative patient and injury factors of successful rehabilitation after anterior cruciate ligament reconstruction with single-bundle techniques. Arthroscopy 2013;29:1879-95.

23 Ericsson $Y B$, Roos EM, Frobell RB. Lower extremity performance following $A C L$ rehabilitation in the KANON-trial: impact of reconstruction and predictive value at 2 and 5 years. Br I Sports Med 2013;47:980-5.

24 Logerstedt D, Lynch A, Axe MJ, et al. Pre-operative quadriceps strength predicts IKDC2000 scores 6 months after anterior cruciate ligament reconstruction. Knee 2013;20:208-12.

25 Shaarani SR, O'Hare C, Quinn A, et al. Effect of prehabilitation on the outcome of anterior cruciate ligament reconstruction. Am J Sports Med 2013;41:2117-27. 